\title{
BMJ Open Effect of combined testing of ceramides with high-sensitive troponin $T$ on the detection of acute coronary syndrome in patients with chest pain in China: a prospective observational study
}

To cite: Yao K, Wang Y, Xu D, et al. Effect of combined testing of ceramides with high-sensitive troponin $\mathrm{T}$ on the detection of acute coronary syndrome in patients with chest pain in China: a prospective observational study. BMJ Open 2019;9:e028211. doi:10.1136/ bmjopen-2018-028211

- Prepublication history and additional material for this paper are available online. To view these files, please visit the journal online (http://dx.doi. org/10.1136/bmjopen-2018028211).

$\mathrm{KY}, \mathrm{YW}$ and $\mathrm{DX}$ contributed equally.

Received 4 December 2018 Revised 24 May 2019 Accepted 30 May 2019

Check for updates

(c) Author(s) (or their employer(s)) 2019. Re-use permitted under CC BY-NC. No commercial re-use. See rights and permissions. Published by BMJ.

For numbered affiliations see end of article.

Correspondence to

Dr Junbo Ge;

ge.junb02@zs-hospital.sh.cn

\section{ABSTRACT}

Objective Ceramides are associated with coronary plaque vulnerability. We aim to investigate the potential diagnostic value of ceramides for acute coronary syndrome (ACS) in Chinese patients with chest pain.

Design Prospective observational survey.

Setting Shanghai, China, 2016-2017.

Participants A total of 2773 patients with chest pain from four hospitals in Shanghai, China, between August 2016 and October 2017.

Main outcome measures Performance of metabolites model in detection of ACS cases including ST-elevation myocardial infarction (STEMI), non-STEMI (NSTEMI) and unstable angina.

Results Plasma levels of 12 ceramide molecules and corresponding ratios were compared between patients diagnosed with ACS and those without. Cer(d18:1/24:1(15Z))/Cer(d18:1/24:0) ratio, Cer(d18:1/14:0) and Cer(d18:1/22:0) were independent predictors of ACS after adjustment of traditional risk factors and high-sensitivecardiac troponin T. Receiver operating characteristic curve analysis showed a significant improvement in detecting ACS in the multivariable model with ceramides compared with that without $(0.865$ ( 0.840 to 0.889$)$ vs 0.808 (0.776 to 0.841$)$, $\mathrm{p}<0.001)$.

Conclusion Distinct plasma ceramides are independent diagnostic predictors of ACS among patients with chest pain. Ceramides together with high-sensitive troponin and traditional factors showed great potential in identifying ACS among patients with chest pain.

\section{INTRODUCTION}

Acute coronary syndrome (ACS), which includes both myocardial infarction (MI) and unstable angina (UA), is estimated to affect 1.4 million people in the USA and 2.5 million people in China per year. ${ }^{12}$ However, patients with acute chest pain suggestive of ACS present with a heterogeneous array of conditions, including both non-ischaemic and

\section{Strengths and limitations of this study}

This is the first study with systematic demonstration of plasma levels of 12 ceramide molecules in 2773 Chinese patients with chest pain.

- Performance of ceramides levels in diagnosing acute coronary syndrome was assessed and validated in multicentre clinical studies.

- The time of ceramides level elevation after acute coronary syndrome (ACS) occurrence cannot be assessed in the study design.

ischaemic chest pain. ${ }^{3}$ Only $17 \%$ of these are finally diagnosed as ACS caused by atherosclerotic and ischaemic heart disease. ${ }^{3}$

The likelihood of ACS in patients with chest pain is estimated via the entire clinical picture, including symptoms and physical examination findings, disease history, ECG changes and biomarkers results. Among biomarkers, cardiac troponins play a central role in establishing a diagnosis and stratifying risk. Troponins are more specific and sensitive than the traditional cardiac enzymes such as creatine kinase (CK), its isoenzyme MB (CK-MB) and myoglobin. ${ }^{4}$ However, there has been an increasing recognition of other biomarkers recently. ${ }^{5}$ Considerable effort has been made to improve the multibiomarker-based evaluation and management of ACS. Altered lipid metabolism associated with inflammation and oxidative stress initiates the pathological changes, including the formation of lipid-laden foam cells and the necrotic lipid core of unstable plaque. ${ }^{67}$ As many lipid types are essential in atherogenesis, they should be used for ACS prediction. ${ }^{6}$

Ceramides are a family of lipid molecules that are found in high concentrations within 
cell membranes and play a key role in a variety of physiological functions including apoptosis, cell growth, cell adhesion and plasma membrane integrity maintenance. ${ }^{8}$ All tissues can synthesise ceramides de novo from saturated fats and sphingosine. ${ }^{9}$ However, lesional low-density lipoprotein (LDL) is known to be rich in ceramide in the atherosclerotic plaque, and it contains 10-fold to 50 -fold higher content of ceramide when compared with plasma LDL. ${ }^{10}$ An arterial-wall sphingomyelinase (SMase) hydrolyzes the sphingomyelin of retained LDL. ${ }^{10}$ Plasma ceramide (d18:1/16:0) is associated with the fraction of necrotic core tissue and lipid core burden in coronary atherosclerosis. ${ }^{11}$ Inflammatory cytokine tumour necrosis factor alpha was shown to induce the production of ceramide in vascular endothelial cells, ${ }^{12} 13$ via the activation of SMase, accompanied by reactive oxygen species production. ${ }^{14}$ High content of oxidised LDL in the plaque is capable of activating precursors to matrix metallopeptidase 2, propagating the signalling of the ceramide pathway by activation of SMase. ${ }^{15}$ These findings suggest abnormal regulation of ceramides might be related to plaque instability and triggering of ACS.

Previous clinical research found that circulating ceramides are elevated in patients with hypertension, ${ }^{16}$ obesity $^{17} 18$ and type II diabetes mellitus. ${ }^{18}$ Ceramides ratio levels such as $\operatorname{Cer}(\mathrm{d} 18: 1 / 16: 0) / \operatorname{Cer}(\mathrm{d} 18: 1 / 24: 0)$ ratio, $\operatorname{Cer}(\mathrm{d} 18: 1 / 18: 0) / \operatorname{Cer}(\mathrm{d} 18: 1 / 24: 0)$ ratio and $\operatorname{Cer}(\mathrm{d} 18: 1 / 24: 1) / \operatorname{Cer}(\mathrm{d} 18: 1 / 24: 0)$ ratio were proposed as useful biomarkers for cardiovascular death (CV death) prediction, as assessed by three independent coronary artery disease (CAD) cohorts. $^{19}$

Although several studies have shown plasma levels of specific ceramide molecules are correlated with diagnosis of patients with cardiac events, no studies to date have evaluated the role of these ceramide molecules in detecting ACS from patients with chest pain. In this study, we aim to assess the value of ceramides in detection of ACS in patients with chest pain and whether a combination of ceramides and troponin could improve the diagnostic power.

\section{METHODS \\ Study design and participants}

This is a prospective observational study involving four University-affiliated hospitals in Shanghai, China. A total of 2990 patients with chest pain were consecutively recruited in chest pain outpatient during Monday to Friday between August 2016 and October 2017, and 2773 patients were finally admitted to wards of cardiology after prescreening by the exclusion criteria. Sample size calculation is shown in Supplementary File. Patients were eligible if they were aged $\geq 18$ and $\leq 80$ years, presented with signs and symptoms of chest pain and agreed to participate in this study. The exclusion criteria were pregnant women, organ transplant patients, patients suffering from bleeding disorders, patients with neoplasms with a life expectancy $<1$ year, chronic kidney disease (estimated glomerular filtration rate $<60 \mathrm{~mL} / \mathrm{min} / 1.73 \mathrm{~m}^{2}$ ) and patients with a clear non-cardiac chest pain. A flowchart describing the inclusion and exclusion process is shown in figure 1. Full study protocol is provided on request to the corresponding author.

The study complies with the Declaration of Helsinki and was approved by the Ethics Committee of Zhongshan Hospital, the Ethics Committee of Tongji Hospital, the

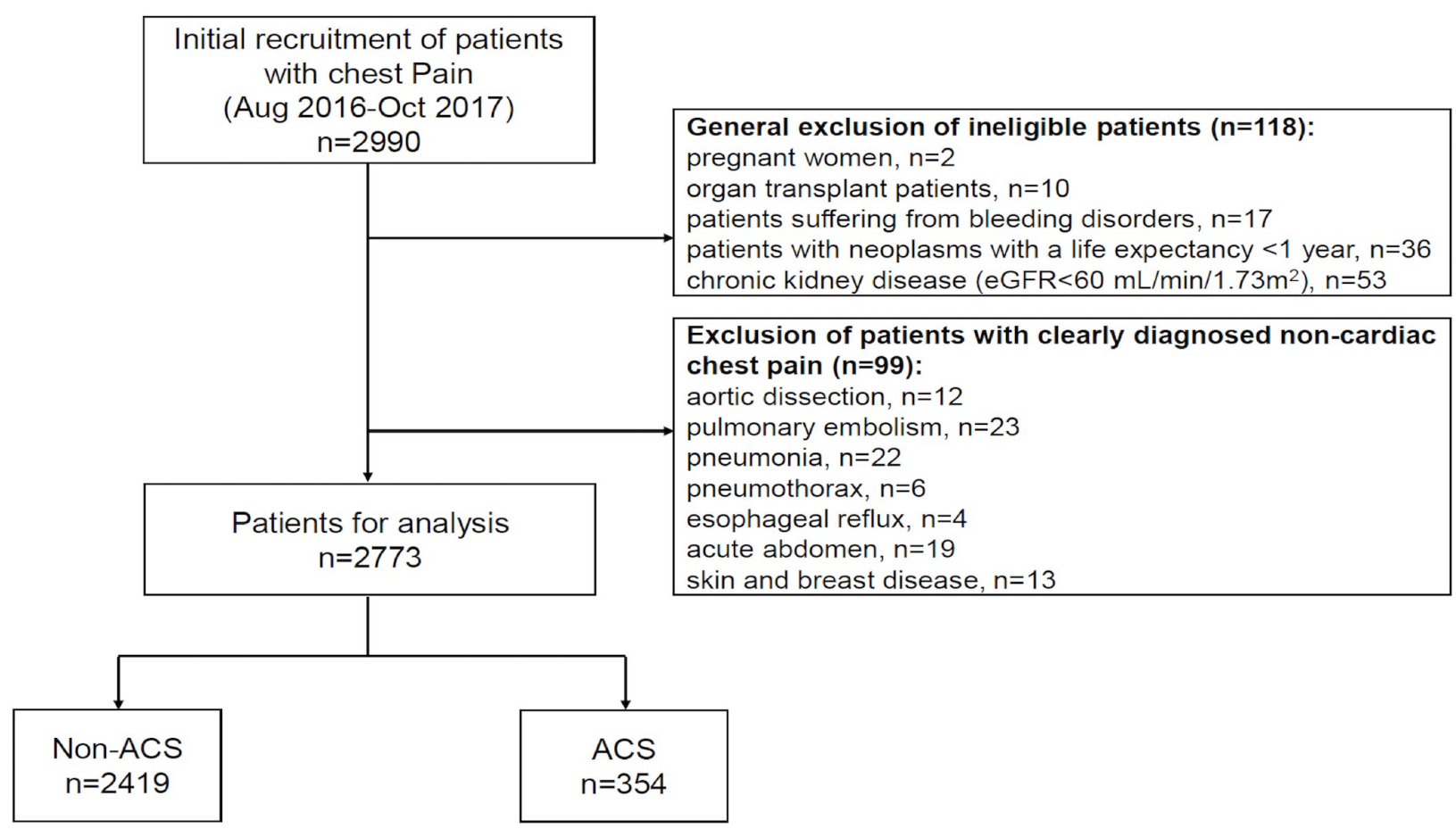

Figure 1 Flowchart. ACS, acute coronary syndrome; eGFR, estimated glomerular filtration rate. 
Table 1 Demographics, clinical and laboratory findings of included patients in total cohort

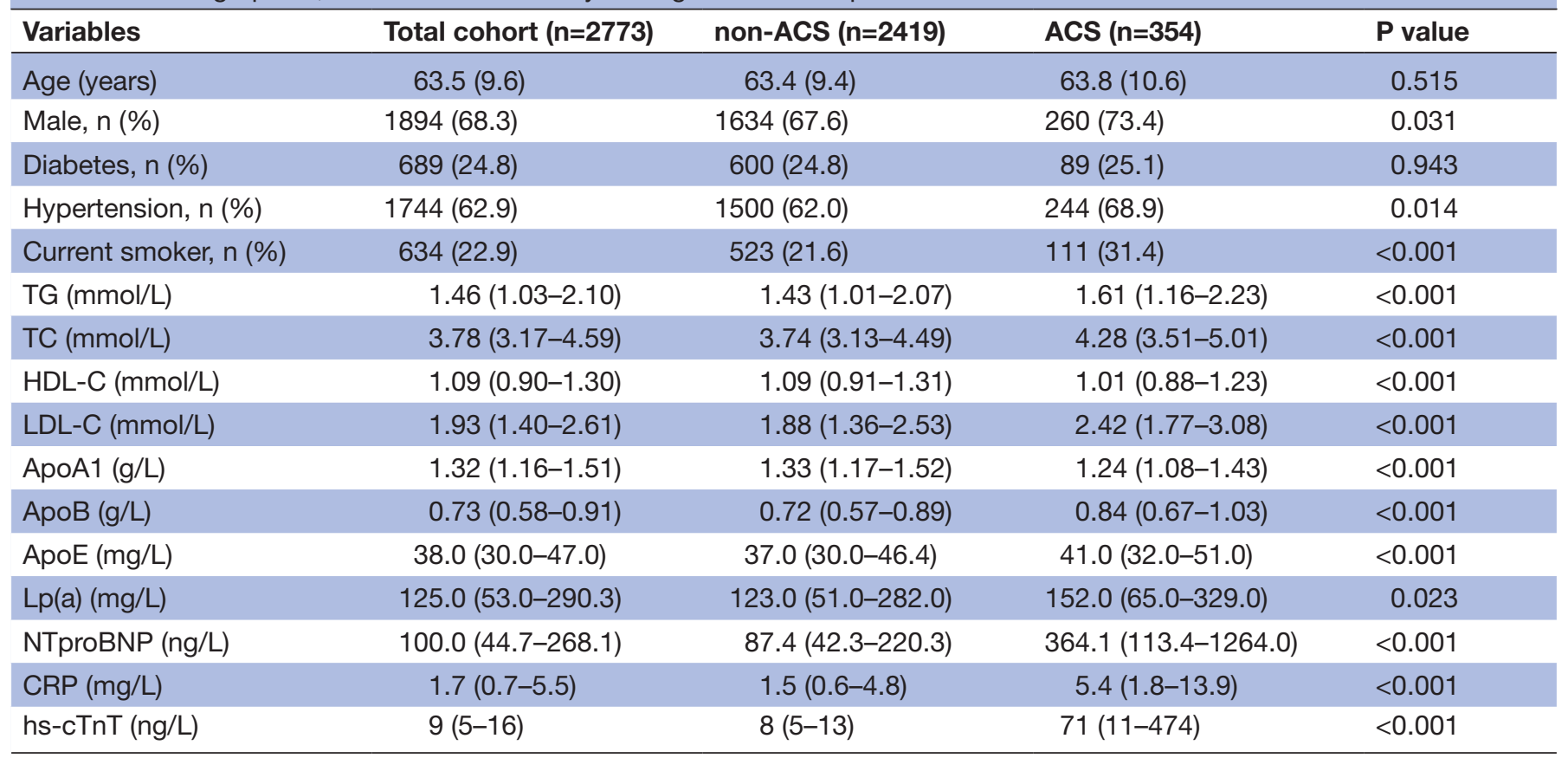

Data are median (IQR) or count (percentage).

$P$ value for the difference between patients diagnosed as ACS and non-ACS.

ACS, acute coronary syndrome ApoA1, apolipoprotein A1; ApoB, apolipoprotein B; ApoE, apolipoprotein E; CRP, C reactive protein; HDL-C, high-density lipoprotein cholesterol; hs-cTnT, high-sensitivity cardiac troponin T; LDL-C, low-density lipoprotein cholesterol; LP(a), lipoprotein(a); NTproBNP, N-terminal pro-brain natriuretic peptide; TC, total cholesterol; TG, triglyceride.

Ethics Committee of Shanghai Jiao Tong University Affiliated Sixth People's Hospital and the Ethics Committee of Minhang Hospital. Written informed consent was obtained from all participants.

\section{Clinical and laboratory data}

Clinical history, physical examination findings and blood tests including renal and liver functions, $\mathrm{C}$ reactive protein (CRP), D-dimer and high-sensitive cardiac troponin T (hs-cTnT; Roche) levels, ECG, coronary angiogram and echocardiogram findings were recorded in all patients. Troponin levels were measured by electrochemiluminescence method using hs-cTnT assay (Roche Diagnostics) on Roche Cobas e601. The coefficient of variation in the hs-cTnT assay is $\leq 10 \%$ at the cut-off value of $13 \mathrm{ng} / \mathrm{L}$. The 99 percentile upper reference limit of hs-cTnT assay is $14 \mathrm{ng} / \mathrm{L}$. Besides, the assay also has a limit of blank of $3 \mathrm{ng} / \mathrm{L}$ and a limit of detection of $5 \mathrm{ng} / \mathrm{L}$, and the analytical range is $3-10000 \mathrm{ng} / \mathrm{L}$. Regular blood lipid tests were conducted using standard methods. Specifically, total cholesterol (TC) was measured by enzymatic cholesterol method using cholesterol oxidase/peroxidase aminophenazone reagent, while total triglyceride (TG) was measured by glycerol-3-phosphate oxidase/peroxidase anti-peroxidase method. LDL cholesterol (LDL-C) and high-density lipoprotein cholesterol (HDL-C) were measured by homogenous polyethylene glycol modification enzyme method. Other blood biomedical assay of apolipoprotein (AI, AII, B and E), lipoprotein (a) and lipoprotein-associated phospholipase were performed by immune transmission turbidity method. Performers of the above tests were blinded to the diagnosis of diseases. The detection limit, analytical range and reference interval or decision limit are summarised in online supplementary table 1 .

\section{Diagnosis of ACS}

The diagnosis of ACS including UA, non-ST segment elevation MI (NSTEMI) and ST-segment elevation MI (STEMI) was made by two independent cardiologists by reviewing all patients' notes, including symptoms, 12-lead ECG and blood tests results according to the 2014 American Heart Association/American College of Cardiology guidelines and international definition of MI version 3. Type 1 and type 2 MI were both included. ${ }^{20} 21$ The agreement level and kappa value between the two cardiologists were 0.99 and 0.98 . The cases where the two cardiologists disagreed were reviewed by a senior cardiologist. All the cardiologists were blinded to ceramide levels. Cardiac chest pain with persistent ECG findings of elevation in the ST segment indicate STEMI. Cardiac chest pain and elevations in troponins levels without ST-elevation indicate NSTEMI. Those with presence of one or more of three principal ischaemic symptoms ((1) rest angina (lasting $>20 \mathrm{~min})$, (2) new-onset $(<2$ months previously) severe angina and (3) a crescendo pattern of occurrence (increasing in intensity, duration, frequency or any combination of these factors)) without elevations in cardiac troponins are defined as UA. ${ }^{5}$ Participants 
Table 2 Plasma levels of ceramides molecules and ratios in total cohort and by ACS status

\begin{tabular}{|c|c|c|c|c|}
\hline Ceramides ( $\mu \mathrm{mol} / \mathrm{L})$ & Total cohort $(n=2773)$ & non-ACS (n=2419) & ACS $(n=354)$ & $P$ value \\
\hline Cer(d18:1/16:0) & $0.203(0.165-0.248)$ & $0.198(0.162-0.241)$ & $0.237(0.203-0.286)$ & $<0.001$ \\
\hline Cer(d18:1/18:0) & $0.051(0.037-0.069)$ & $0.049(0.036-0.065)$ & $0.068(0.051-0.084)$ & $<0.001$ \\
\hline Cer(d18:1/24:1(15Z)) & $0.583(0.448-0.767)$ & $0.569(0.436-0.739)$ & $0.730(0.557-0.950)$ & $<0.001$ \\
\hline Cer(d18:1/14:0) & $0.0027(0.0020-0.0036)$ & $0.0027(0.0020-0.0035)$ & $0.0030(0.0024-0.0040)$ & $<0.001$ \\
\hline Cer(d18:1/22:0) & $0.381(0.300-0.490)$ & $0.375(0.294-0.480)$ & $0.416(0.336-0.540)$ & $<0.001$ \\
\hline Cer(d18:0/16:0) & $0.010(0.007-0.014)$ & $0.010(0.007-0.013)$ & $0.011(0.008-0.015)$ & $<0.001$ \\
\hline Cer(d18:0/18:0) & $0.005(0.003-0.008)$ & $0.005(0.003-0.007)$ & $0.007(0.004-0.011)$ & $<0.001$ \\
\hline Cer(d18:0/24:0) & $0.061(0.042-0.086)$ & $0.060(0.042-0.086)$ & $0.068(0.048-0.094)$ & $<0.001$ \\
\hline Cer(d18:0/24:1(15Z)) & $0.029(0.020-0.044)$ & $0.028(0.019-0.041)$ & $0.040(0.026-0.058)$ & $<0.001$ \\
\hline Cer(d18:1/18:0)/Cer(d18:1/24:0) & $0.024(0.019-0.032)$ & $0.023(0.018-0.031)$ & $0.031(0.023-0.039)$ & $<0.001$ \\
\hline Cer(d18:1/24:1(15Z))/Cer(d18:1/24:0) & $0.269(0.214-0.360)$ & $0.261(0.211-0.345)$ & $0.321(0.253-0.426)$ & $<0.001$ \\
\hline
\end{tabular}

Data are median (IQR).

$P$ value for the difference between patients diagnosed as ACS and non-ACS.

ACS, acute coronary syndrome.

were diagnosed with ACS when there is evidence of UA, NSTEMI or STEMI.

\section{Quantification of plasma ceramides}

Potential ceramides were generated from previous untargeted and targeted ceramide profile studies. ${ }^{22}{ }^{23}$ Analyses of 12 plasma ceramides were performed using a Waters Xevo TQ-S mass spectrometer (Manchester, UK) equipped with Waters Acuity UPLC I-Class (Milford, Massachusetts, USA) in a hospital and Qlife lab collaborative laboratory based in Department of Cardiology. The mass spectrometry was operated in multiple-reaction monitoring mode with electro spray ionisation interface (ESI)-positive ionisation. The capillary voltage was set at $3.0 \mathrm{kV}$, and the source temperature was $120^{\circ} \mathrm{C}$. The desolvation temperature and gas flow were $400^{\circ} \mathrm{C}$ and $800 \mathrm{~L} / \mathrm{h}$, respectively. The source offset was maintained at $60 \mathrm{~V}$.

Blood samples for ceramides test were collected and centrifuged using EDTA anticoagulation tube at admission. Plasma were immediately stored in $-80^{\circ} \mathrm{C}$ for future analysis. Ceramides tests were taken immediately after samples of all patients were collected. Before analysis, the samples were thawed at room temperature, then a volume of $800 \mu \mathrm{L}$ of protein precipitation solution (isopropanol) that containing D7-Cer d18:1/16:0 $(0.01 \mathrm{pmol} / \mu \mathrm{L}), \quad \mathrm{D} 7-\mathrm{Cer} \quad \mathrm{d} 18: 1 / 18: 0 \quad(0.005 \mathrm{pmol} /$ $\mu \mathrm{L})$, D7-Cer d18:1/24:0 (0.015 pmol/ $\mu \mathrm{L})$ and D7-Cer $\mathrm{d} 18: 1 / 24: 1(0.015 \mathrm{pmol} / \mu \mathrm{L})$ was pipetted into $1.5 \mathrm{~mL}$ Eppendorf tube after addition of $50 \mu \mathrm{L}$ of plasma sample. The mixture was thoroughly vortexed for $10 \mathrm{~min}$ followed by 5-min centrifugation at $14000 \mathrm{rpm}$. Supernatant $(70 \mu \mathrm{L})$ was collected for liquid chromatography-mass spectrometry (LC/MS/MS) analysis. Ceramides ratios were calculated using $\operatorname{Cer}(\mathrm{d} 18: 1 / 16: 0), \operatorname{Cer}(\mathrm{d} 18: 1 / 18: 0)$, Cer(d18:1/24:1(15Z)) and Cer(d18:1/24:0) ${ }^{23}$ Lab technicians who were in charge of ceramides test were blinded to disease diagnosis.

\section{Statistical methods}

Continuous variables are summarised as mean (SD) or median (IQR) and categorical data as count (percentage). Student's t-test, Mann-Whitney U and Wilcoxon signed rank tests were used to test differences in continuous variables where appropriate, and $\chi^{2}$ test was used for proportions. Univariate logistic regression was used to analyse the association of each variable and binary outcome. Multivariable logistic regression analyses were undertaken to identify the demographic, clinical and laboratory factors associated with ACS in patients with chest pain after adjusting for potential confounders, and the final models included variables significantly associated with ACS.

Training and validation cohorts were developed via a 10 -fold cross-validation approach repeated 10 times. $^{24}$ The estimate of prediction error is almost unbiased under 10-fold cross-validation. Repeated 10 times that minimise the cross-validated error is then used to build the final model. ${ }^{2526}$ The performance and discrimination ability of the five models were assessed using the area under the receiver operating curve (AUROC) and net reclassification index (NRI). ROC curves and NRI values were compared among models in training and validation datasets. 
All tests were two-tailed, and $\mathrm{p}$ values $<0.05$ were considered statistically significant. OR with $95 \%$ CIs for the chosen clinical and laboratory variables in the logistic regression models, and AUROC and NRI values with $95 \%$ CIs for different test datasets were calculated. Statistical analyses were done with statistical software R V.3.4.3.

\section{Patient and public involvement}

This study was conducted without patient and public involvement.

\section{RESULTS \\ Demographics, lipid profiles and laboratory findings of patients}

The demographics, clinical and laboratory findings of all participants are reported in table 1 . Serial troponin levels were measured at $0-3 \mathrm{~h}$ after admission in all recruited patients, and all patients underwent echocardiography and coronary angiography. In 2773 patients with chest pain, $354(12.8 \%)$ were diagnosed with ACS, among whom 116 (4.2\%) were UA, 114 (4.1\%) were NSTEMI and $124(4.5 \%)$ were STEMI. All 116 patients with UA had a significant stenosis on coronary angiography. Compared with those without ACS, patients with ACS are more likely to be men $(73.44 \%$ vs $67.57 \%)$, to be current smokers (31.35\% vs $21.62 \%)$ and to have hypertension $(68.92 \%$ vs $62.00 \%)$. Lipid profiles and lipoproteins including TG, TC, LDL-C, ApoB, ApoE and lipoprotein(a) (Lp(a)) were significantly elevated in patients diagnosed with ACS, while HDL-C and apolipoprotein A1 (ApoA1) were significantly lower in ACS patients (all $\mathrm{p}<0.001$, except for $\operatorname{Lp}(a), p=0.023)$. CRP and cardiac damage markers including hs-cTnT and N-terminal pro-brain natriuretic peptide (NTproBNP) levels were significantly higher in ACS patients (all $\mathrm{p}<0.001)$. Demographics, lipid profiles and laboratory findings of patients diagnosed with STEMI, NSTEMI and UA were also reported in online supplementary table 2 .

\section{Plasma ceramides levels in ACS patients}

Table 2 presents the levels of 12 ceramides molecules in patients with and without ACS. The levels of 12 ceramide molecules (Cer(d18:1/16:0), $\operatorname{Cer}(\mathrm{d} 18: 1 / 18: 0)$, Cer(d18:1/24:0), $\quad \operatorname{Cer}(\mathrm{d} 18: 1 / 24: 1(15 \mathrm{Z}))$, Cer(d18:1/14:0), Cer(d18:1/20:0), Cer(d18:1/22:0), $\operatorname{Cer}(d 18: 0 / 16: 0), \quad \operatorname{Cer}(\mathrm{d} 18: 0 / 18: 0), \quad \operatorname{Cer}(\mathrm{d} 18: 0 / 24: 0)$, Cer(d18:0/24:1(15Z)) and Cer(d18:1/24:1)) were all significantly elevated in patients with ACS compared with those without (all $\mathrm{p}<0.001$, except for $\operatorname{Cer}(\mathrm{d} 18: 1 / 24: 0)$, $\mathrm{p}=0.028 ;$ table 2$)$. In addition, three ceramide ratios (Cer(d18:1/16:0)/Cer(d18:1/24:0), Cer(d18:1/18:0)/ $\operatorname{Cer}(d 18: 1 / 24: 0) \quad$ and $\quad \operatorname{Cer}(d 18: 1 / 24: 1(15 Z)) /$ Cer (d18:1/24:0) ) were also significantly increased in ACS patients with all $\mathrm{p}$ values $<0.001$. In patients diagnosed with UA and NSTEMI, levels of ceramides and ceramide ratios were also significantly higher compared with
Table 3 Univariate logistic regression showing significant predictors of ACS in patients with chest pain

\begin{tabular}{|c|c|c|}
\hline \multirow[b]{2}{*}{ Variables } & \multicolumn{2}{|c|}{ Total cohort $(n=2773)$} \\
\hline & OR $(95 \% \mathrm{Cl})$ & $P$ value \\
\hline Age & 1.00 (0.99 to 1.02$)$ & 0.476 \\
\hline Gender & 1.33 (1.04 to 1.71$)$ & 0.027 \\
\hline Diabetes & $1.02(0.78$ to 1.31$)$ & 0.890 \\
\hline Hypertension & 1.36 (1.07 to 1.73$)$ & 0.012 \\
\hline Current smoker & 1.66 (1.29 to 2.11$)$ & $<0.001$ \\
\hline $\mathrm{TG}^{*}$ & 1.40 (1.15 to 1.70$)$ & $<0.001$ \\
\hline $\mathrm{TC}^{*}$ & 5.17 (3.30 to 8.15$)$ & $<0.001$ \\
\hline HDL-C* & 0.44 (0.30 to 0.66$)$ & $<0.001$ \\
\hline LDL-C* & 3.14 (2.37 to 4.17 ) & $<0.001$ \\
\hline $\mathrm{ApoA} 1^{*}$ & 0.17 (0.09 to 0.29$)$ & $<0.001$ \\
\hline $\mathrm{ApoB}^{\star}$ & 2.83 (2.05 to 4.01$)$ & $<0.001$ \\
\hline $\mathrm{ApoE}^{*}$ & 0.93 (0.76 to 1.17$)$ & 0.534 \\
\hline $\operatorname{Lp}(a)^{\star}$ & 1.10 (1.00 to 1.21$)$ & 0.055 \\
\hline NTproBNP* & 1.73 (1.61 to 1.88$)$ & $<0.001$ \\
\hline $\mathrm{CRP}^{\star}$ & 1.74 (1.59 to 1.90$)$ & $<0.001$ \\
\hline hs-cTnT* & 2.45 (2.24 to 2.70$)$ & $<0.001$ \\
\hline Cer(d18:1/16:0)* & $7.53(5.11$ to 11.17$)$ & $<0.001$ \\
\hline Cer(d18:1/18:0)* & 4.67 (3.55 to 6.17$)$ & $<0.001$ \\
\hline Cer(d18:1/24:0)* & 1.41 (1.05 to 1.90$)$ & 0.025 \\
\hline $\operatorname{Cer}(\mathrm{d} 18: 1 / 24: 1(15 Z))^{*}$ & 5.09 (3.79 to 6.87$)$ & $<0.001$ \\
\hline Cer(d18:1/14:0)* & 2.04 (1.59 to 2.62 ) & $<0.001$ \\
\hline Cer(d18:1/20:0)* & 4.55 (3.35 to 6.22$)$ & $<0.001$ \\
\hline Cer(d18:1/22:0)* & 2.36 (1.75 to 3.18$)$ & $<0.001$ \\
\hline Cer(d18:0/16:0)* & 2.02 (1.61 to 2.55$)$ & $<0.001$ \\
\hline $\operatorname{Cer}(d 18: 0 / 18: 0)^{\star}$ & 2.26 (1.92 to 2.66$)$ & $<0.001$ \\
\hline Cer(d18:0/24:0)* & 1.49 (1.21 to 1.84$)$ & $<0.001$ \\
\hline Cer(d18:0/24:1(15Z))* & 2.73 (2.24 to 3.33$)$ & $<0.001$ \\
\hline $\operatorname{Cer}(\mathrm{d} 18: 1 / 24: 1)^{\star}$ & 1.57 (1.26 to 1.96$)$ & $<0.001$ \\
\hline Cer(d18:1/16:0)/Cer(d18:1/24:0)* & 4.11 (2.87 to 5.89$)$ & $<0.001$ \\
\hline Cer(d18:1/18:0)/Cer(d18:1/24:0)* & 3.84 (2.93 to 5.05$)$ & $<0.001$ \\
\hline Cer(d18:1/24:1(15Z))/Cer(d18:1/24:0)* & 2.63 (2.08 to 3.34$)$ & $<0.001$ \\
\hline
\end{tabular}

Data are OR $(95 \% \mathrm{Cl})$.

*Log transformed before analysis.

ACS, acute coronary syndrome; ApoA1, apolipoprotein A1; ApoB apolipoprotein B; ApoE, apolipoprotein E; Cer, ceramides; CRP, C reactive protein; HDL-C, high-density lipoprotein cholesterol; hs-cTnT, highsensitivity cardiac troponin T; LDL-C, low-density lipoprotein cholesterol; LP(a), lipoprotein(a); NTproBNP, N-terminal pro-brain natriuretic peptide; TC, total cholesterol; TG, triglyceride.

patients diagnosed with non-ACS (see online supplementary table 3$)$.

\section{Association between plasma ceramides and traditional and cardiac damage markers}

The associations between plasma ceramides with traditional ACS risk factors and cardiac markers for myocardial injury are shown in online supplementary table 4 . Most of the 12 ceramide molecules and ceramide ratios were moderately correlated with lipid profiles including TG, TC, LDL-C, ApoB and ApoE, while CRP was shown to be weakly correlated with ceramides. There was no 
Table 4 Multivariable logistic regression showing significant predictors of ACS in patients with chest pain after adjustment for conventional risk factors

\begin{tabular}{|c|c|c|c|c|c|}
\hline Baseline variables & Model 1 & Model 2 & Model 3 & Model 4 & Model 5 \\
\hline Hypertension & $1.37(1.03 \text { to } 1.83)^{*}$ & - & 1.35 (0.98 to 1.87$)$ & $1.41(1.06 \text { to } 1.90)^{*}$ & $1.40(1.01 \text { to } 1.96)^{*}$ \\
\hline Current smoker & $1.93(1.43 \text { to } 2.58)^{\star \star \star}$ & - & $1.52(1.09 \text { to } 2.10)^{*}$ & $1.99(1.47 \text { to } 2.69)^{\star * *}$ & $1.58(1.12 \text { to } 2.21)^{\star \star}$ \\
\hline CRP† & $1.40(1.27 \text { to } 1.55)^{\star \star \star}$ & - & $1.20(1.07 \text { to } 1.34)^{\star \star}$ & $1.38(1.25 \text { to } 1.53)^{\star \star \star}$ & $1.16(1.03 \text { to } 1.31)^{\star}$ \\
\hline NTproBNP† & $1.63(1.49 \text { to } 1.78)^{\star \star \star}$ & - & $1.16(1.04 \text { to } 1.29)^{\star \star}$ & $1.57(1.44 \text { to } 1.72)^{\star \star \star}$ & 1.07 (0.95 to 1.19$)$ \\
\hline Cer(d18:1/14:0)† & - & - & - & $1.44(1.01 \text { to } 2.06)^{\star}$ & $1.74(1.17 \text { to } 2.61)^{\star *}$ \\
\hline Cer(d18:1/22:0)† & - & - & - & $2.08(1.32 \text { to } 3.29)^{\star \star}$ & $2.30(1.37 \text { to } 3.87)^{\star *}$ \\
\hline
\end{tabular}

Data are OR $(95 \% \mathrm{Cl})$.

Model 1. Included traditional risk factors (hypertension, current smoker, LDL-C, CRP and NTproBNP levels).

Model 2. Only Included hs-cTnT.

Model 3. Included traditional risk factors and hs-cTnT.

Model 4. Included traditional risk factors and ceramides.

Model 5. Included traditional risk factors, hs-cTnT and ceramides.

${ }^{*} \mathrm{P}<0.05,{ }^{* *} \mathrm{p}<0.01,{ }^{* * *} \mathrm{p}<0.001$.

†Log transformed before analysis.

ACS, acute coronary syndrome; Cer, ceramides; CRP, C reactive protein; hs-cTnT, high-sensitivity cardiac troponin T; LDL-C, low-density

lipoprotein cholesterol; NTproBNP, N-terminal pro-brain natriuretic peptide.

significant correlation between ceramides and myocardial damage markers including hs-cTnT and NTproBNP.

\section{Clinical predictors and multivariable models for ACS}

Clinical predictors for ACS were identified in univariate logistic regression analysis and are shown in table 3. Being male, having hypertension and being current smoker were significantly associated with ACS $(\mathrm{p}<0.05)$. Besides, most of the lipid profile (TG, TC, HDL-C, LDL-C, ApoA1 and ApoB) were significantly associated with ACS (all $\mathrm{p}<0.001$ ). Also, increased levels of hs-cTnT (OR and 95\% CI: 2.45 (2.23 to 2.70)), NTproBNP (1.73 (1.60 to 1.87)) and CRP (1.73 (1.58 to 1.90$)$ ) were significantly associated with ACS. Furthermore, all 12 ceramides levels and three ceramides ratios were significantly associated with ACS with all $\mathrm{p}<0.001$ except Cer(d18:1/24:0) with $\mathrm{p}=0.025$ (table 3).
Multivariable logistic regression analysing independently significant predictors of ACS are shown in table 4. Model 1 included traditional risk factors such as hypertension, current smoker, CRP, LDL-C and NTproBNP (all $\mathrm{p}<0.001$ except for hypertension $\mathrm{p}=0.033$ ). Model 2 only included hs-cTnT (2.454 (2.239 to 2.701), p<0.001). Model 3 included traditional risk factors and hs-cTnT. Current smoker, CRP, LDL-C, NTproBNP as well as hs-cTnT were independently significant in detecting patients with ACS. Model 4 included traditional risk factors and ceramides levels, with OR (95\% CI) of Cer(d18:1/24:1(15Z))/Cer(d18:1/24:0): 2.960 (2.156 to 4.072), $\operatorname{Cer}(\mathrm{d} 18: 1 / 14: 0): 1.439$ (1.007 to 2.062$)$ and Cer(d18:1/22:0): 2.079 (1.315 to 3.288). Model 5 included traditional risk factors, hs-cTnT $(2.190,(1.939$ to 2.485$))$ and ceramides levels $(\operatorname{Cer}(\mathrm{d} 18: 1 / 24: 1(15 \mathrm{Z})) / \operatorname{Cer}(\mathrm{d} 18: 1 / 24: 0)$ :

Table 5 Discrimination and reclassification of ACS with various multivariable logistic regression models

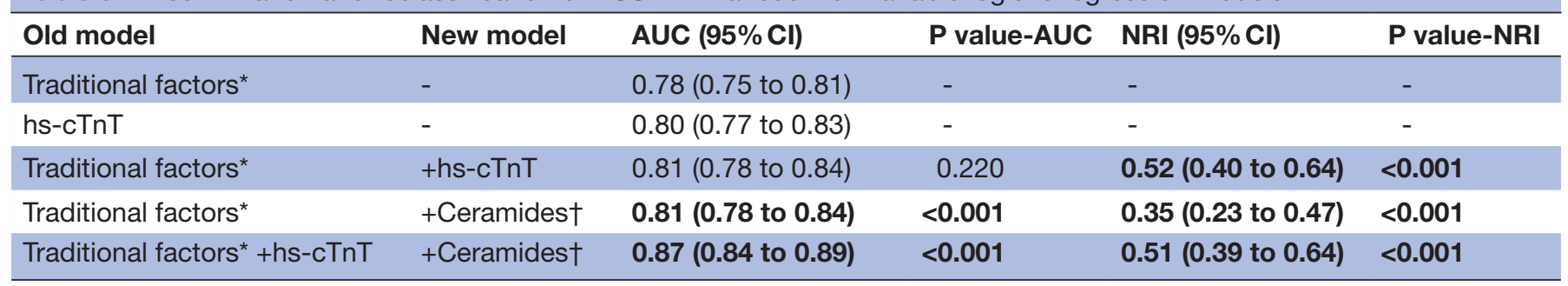

$P$ values AUC and $p$ values NRI are for the difference between old models and new models. Bold used to highlight those $p$ values $<0.05$.

*Traditional risk factors include hypertension, current smoker, LDL-C, CRP and NTproBNP levels.

†Ceramides include Cer(d18:1/24:1(15Z)/Cer(d18:1/24:0), Cer(d18:1/14:0) and Cer(d18:1/22:0).

LDL-C, CRP, NT-proBNP, hs-cTnT and ceramides levels are log transformed before analysis.

ACS, acute coronary syndrome; CRP, C reactive protein; hs-cTnT, high-sensitivity cardiac troponin T; LDL-C, low-density lipoprotein cholesterol; NT-proBNP, N-terminal pro-brain natriuretic peptide. 

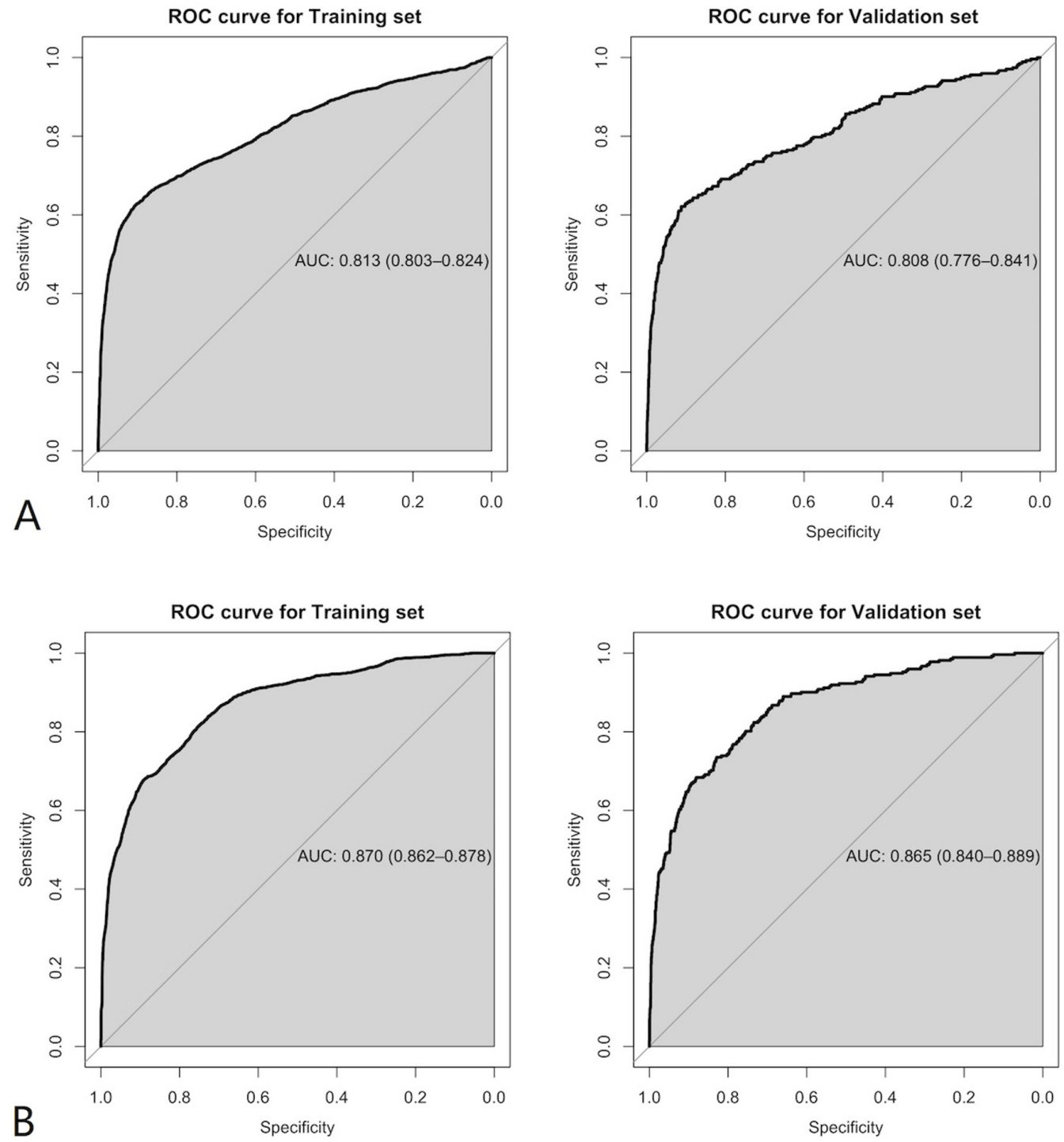

Figure 2 Receiver operating characteristic (ROC) curves of multivariable models for the discrimination of ACS. (A) Traditional risk factors (hypertension, current smoker, LDL-C, CRP and NT-proBNP) and high-sensitive troponin are included in the model. (B) Traditional risk factors (hypertension, current smoker, LDL-C, CRP and NT-proBNP), high-sensitive troponin, Cer(d18:1/14:0), $\operatorname{Cer}(\mathrm{d} 18: 1 / 22: 0)$ and $\operatorname{Cer}(\mathrm{d} 18: 1 / 24: 1(15 \mathrm{Z})) / \operatorname{Cer}(\mathrm{d} 18: 1 / 24: 0)$ are included in the model. ACS, acute coronary syndrome; AUC, area under the curve; CRP, C reactive protein; LDL-C, low-density lipoprotein cholesterol; NT-proBNP, N-terminal pro-brain natriuretic peptide .

4.455 (3.112 to 6.396$), \operatorname{Cer}(\mathrm{d} 18: 1 / 14: 0): 1.73$ (1.166 to 2.605$)$ and $\operatorname{Cer}(\mathrm{d} 18: 1 / 22: 0)$ : 2.301 (1.371 to 3.866$)$, all $\mathrm{p}<0.01)$. Ceramides were shown to be independently associated with ACS after adjusting for traditional factors and hs-cTnT.

\section{Discrimination and reclassification of ACS with various multivariable logistic regression models}

As shown in table 5, the model including traditional factors (hypertension, current smoker, LDL-C, CRP and NT-proBNP level) had an area under the curve (AUC) of 0.780 (0.750 to 0.811) in diagnosing ACS. AUC of hs-cTnT in predicting ACS was 0.800 (0.767 to 0.833 ). After combining traditional factors and hs-cTnT, the AUC became 0.808 ( 0.776 to 0.841 ) (figure $2 \mathrm{~A}$ ). The AUC of model including traditional factors and ceramides was 0.811 ( 0.784 to 0.837 ). Notably, when combining traditional factors, hs-cTnT and ceramides, the AUC of the model rose to 0.865 (0.840 to 0.889 ) (figure $2 \mathrm{~B}$ ). We also compared discrimination and reclassification power of these models. Results showed that ceramides significantly improved the AUC of the model with traditional factors and hs-cTnT (0.865 (0.840 to 0.889$)$ vs 0.808 $(0.776$ to 0.841$), \mathrm{p}<0.001)$. NRI analyses also showed that the model including traditional factors and hs-cTnT was significantly improved by the inclusion of ceramides $(\mathrm{NRI}=0.511(0.388$ to 0.635$), \mathrm{p}<0.001)$.

\section{DISCUSSION}

In this study of 2773 Chinese patients with chest pain from four hospitals in Shanghai, China, levels of 12 ceramides were systematically evaluated together with that of traditional risk factors and high-sensitive troponin. 
Ceramides and their ratios were shown to be independent predictors of ACS in patients with chest pain after adjustment for traditional risk factors (eg, age, sex, body mass index, smoking status and blood cholesterol) and cardiac damage biomarkers (ie, high-sensitive troponin). The proposed model with ceramides showed substantial promise and improved value as a risk evaluation tool for ACS with improved performance on model discrimination and reclassification. With targeted LC/MS/MS-based lipidomic approach, we have successfully established the characteristics of cardiac risk-related ceramides from 2773 Chinese patients with chest pain and confirmed our hypothesis that plasma levels of ceramide subspecies correlate with atherosclerotic plaque instability and hence might be used as lipidomic markers for ACS.

This is to our knowledge the first study with systematic demonstration of plasma levels of 12 ceramide molecules in a Chinese population. Also, the selection of two ceramide molecules and one ceramide ratio as potential biomarkers for ACS has not been reported before. Although our ceramides-based diagnostic model showed great potential in identifying ACS among patients with chest pain, its clinical utility especially regarding rule-in and rule-out strategies and performances still need to be further investigated and validated to make it fully applicable in clinical settings. We were also restricted for not measuring ceramides levels in a serial pattern and lacking information about the relationships between ceramides levels and time from the onset of ACS. The study was possibly also limited by a potential selection bias for patients with higher risk of ACS during recruitment process from admitted patients rather than patients in emergency departments. In addition, the results found in our study need to be validated in independent external cohorts. An improvement in the test technique of ceramides, including standardisation of test protocol and automated pretreatment of samples, is also needed to fulfil the requirement of clinical practice.

Our findings of two ceramides molecules $(\operatorname{Cer}(\mathrm{d} 18: 1 / 14: 0), \operatorname{Cer}(\mathrm{d} 18: 1 / 22: 0))$ and one ceramide ratio $(\operatorname{Cer}(\mathrm{d} 18: 1 / 24: 1(15 \mathrm{Z})) / \operatorname{Cer}(\mathrm{d} 18: 1 / 24: 0))$ as diagnostic biomarkers for ACS among patients with suspected symptoms have never been reported before. In addition, only relatively modest correlations between ceramides and lipid profiles including TG, TC, LDL-C, ApoB and ApoE were detected, and there was no significant correlation between ceramides with hs-cTnT, NT-proBNP and CRP levels, suggesting that the diagnostic value of ceramides for ACS might be independent of the above laboratory variables. Moreover, AUROC of multivariable models showed significant improvement of ceramides on traditional risk factors and high-sensitive troponin $\mathrm{T}$. This result provides evidence that distinct ceramide species serve as independent predictors for the risk of ACS, in addition to conventional blood biomarkers such as HDL-C and CRP levels. Ceramides measurement in highthroughput quality-controlled environments is straightforward. ${ }^{19}$ By setting up clinical laboratories equipped with robotised sample handling systems and mass spectrometry equipment, it would be feasible to identify patients with chest pain at high cardiovascular risk using our ceramides-based diagnostic model. ${ }^{19}$

Previous clinical research found that elevated ceramide plasma concentrations are associated with coronary plaque vulnerability evaluated by intravascular ultrasound virtual histology and near-infrared spectroscopy. ${ }^{11}$ Untargeted lipidomic analyses have identified CAD risk-related ceramide molecules (Cer(d18:1/16:0), Cer(d18:1/18:0) and $\operatorname{Cer}(\mathrm{d} 18: 1 / 24: 1))$ and their ratios with $\operatorname{Cer}(\mathrm{d} 18: 1 / 24: 0)$ as potential risk stratifiers for $\mathrm{CAD}$ patients. ${ }^{22} \mathrm{~A}$ previous study applying ceramides to the prediction of $\mathrm{CV}$ death from patients with $\mathrm{CAD}$ showed that ceramides, independent of other lipid markers and CRP, were significantly associated with CV death. ${ }^{19}$ In a recent study including 495 participants with coronary angiography and followed up for 18 years, ceramides were not associated with $\mathrm{CAD}$ but were independently associated with major adverse cardiovascular events. ${ }^{23}$ A weighted sum of ceramides including Cer22:0 and Cer24:1 was also reported to be associated with a 2.18fold higher risk of cardiovascular disease, which was similar with our results. ${ }^{27}$ Recent studies showed targeted profiling of ceramides predicted short-term and long-term major adverse cardiac events. ${ }^{28}{ }^{29}$ Moreover, a consistent increase in ceramide levels and overexpression of three enzymes in ceramide biosynthesis were found on rat ischaemic myocardium, which is consistent with the elevated plasma levels of ceramides found in our cohort. ${ }^{29}$ Nevertheless, no study has identified the diagnostic value of ceramides in patients with chest pain, while we develop models with lipidomic markers for ACS with additional value on conventional factors and troponin.

Currently, the standard process for the diagnostic assessment of ACS in patients with suspected symptoms relies on clinical evaluation and high-sensitive troponin tests. ${ }^{40}$ However, high-sensitive troponin assays identify a larger number of patients with elevated troponin results but without a final diagnosis of ACS, making interpretation of test results challenging. ${ }^{30}$ The adoption of highly sensitive assays for ACS detection should be accompanied by serial tests and implementation of algorithms, leading to a longer period of observation. ${ }^{31}$ In the current settings of Chinese hospitals, self-paying patients with chest pain tend to be overprescribed by cardiologists with invasive and expensive tests such as coronary angiography. Noteworthily, ceramides levels of patients with UA are higher than those without ACS, which indicates that ceramides may help in identifying patients with UA and higher risk of ACS from populations without significant elevation of cardiac troponins. Moreover, ceramides levels were higher in NSTEMI patients compared with non-ACS ones, indicating a possible role of ceramides in distinguishing between patients with elevated troponins caused by ACS or non-ACS conditions. Nevertheless, the potential roles of ceramides in clinical diagnosis of UA and NSTEMI need further independent investigation and validation. 
In conclusion, we have identified ceramides as independent biomarkers associated with ACS and developed a ceramides-based model to evaluate ACS risk among patients with chest pain. The model showed good model discrimination and net reclassification improvement compared with other models. Our study characterises ceramides species in Chinese patients with chest pain and demonstrates that ceramides are of diagnostic value and have the potential for detecting ACS, in addition to currently used lipid markers and high-sensitive troponin.

\section{Author affiliations}

${ }^{1}$ Department of Cardiology, Zhongshan Hospital, Fudan University. Shanghai Institute of Cardiovascular Diseases, Shanghai, China

${ }^{2}$ Department of Cardiology, Xiamen Branch, Zhongshan Hospital Fudan University, China

${ }^{3}$ School of Population Health and Environmental Sciences, Kings College London, London, UK

${ }^{4}$ Department of Cardiac Surgey, Zhongshan Hospital, Fudan University, Shanghai, China

${ }^{5}$ Department of Cardiac Surgey, Xiamen Branch, Zhongshan HospitalFudan University, Shanghai, China

${ }^{6}$ Department of Cardiology, Tongji Hospital, Tongji University, Shanghai, China ${ }^{7}$ Department of Cardiology, Shanghai Jiao Tong University Affiliated Sixth People's Hospital, Shanghai, China

${ }^{8}$ Department of Cardiology, Minhang Hospital, Fudan University, Shanghai, China

${ }^{9}$ Shanghai Institute of Biomedical Science, Fudan University, Shanghai, China

${ }^{10}$ Qlife Lab Co., Ltd., Shanghai, China

Acknowledgements The authors thank Qlife lab for technical assistance. Contributors JG, KY, YW and DX contributed to study concept and design. KY, $\mathrm{XL}, \mathrm{CS}, \mathrm{WH}, \mathrm{ZW}, \mathrm{RW}, \mathrm{XT}, \mathrm{AS}, \mathrm{YZ}$ and $\mathrm{JQ}$ contributed to recruitment of patients and acquisition of samples. YW, GW, XG and XC contributed to analysis and interpretation of data. GW and YW drafted the first version of the manuscript. All authors contributed to critical revision of the manuscript and approval of final version.

Funding This work was supported by National Key Research and Development Programme (No. 2016YFC1301200) and National Natural Science Foundation of China (No. 81470386).

Competing interests $Q$-life lab holds patents for the diagnostic use of ceramides for cardiovascular risk determination in China. WG, GX and CX work for Q-life and $\mathrm{GX}$ and $\mathrm{CX}$ are also shareholders.

Patient consent for publication Obtained.

Ethics approval This study was approved by the Ethics Committee of Zhongshan Hospital, the Ethics Committee of Tongji Hospital, the Ethics Committee of Shanghai Jiao Tong University Affiliated Sixth People's Hospital and the Ethics Committee of Minhang Hospital.

Provenance and peer review Not commissioned; externally peer reviewed.

Data sharing statement Data are available with the corresponding author and will be available on request.

Open access This is an open access article distributed in accordance with the Creative Commons Attribution Non Commercial (CC BY-NC 4.0) license, which permits others to distribute, remix, adapt, build upon this work non-commercially, and license their derivative works on different terms, provided the original work is properly cited, appropriate credit is given, any changes made indicated, and the use is non-commercial. See: http://creativecommons.org/licenses/by-nc/4.0/.

\section{REFERENCES}

1. Mozaffarian D, Benjamin EJ, Go AS, et al. Heart Disease and Stroke Statistics-2016 Update: A Report From the American Heart Association. Circulation 2016;133:e38-60.

2. Weiwei $C$, Runlin G, Lisheng L, et al. Outline of the report on cardiovascular diseases in China, 2014. Eur Heart J Suppl 2016;18(Suppl F):F2-F11.
3. Pope JH, Aufderheide TP, Ruthazer R, et al. Missed diagnoses of acute cardiac ischemia in the emergency department. N Engl J Med 2000;342:1163-70.

4. Hamm CW, Bassand JP, Agewall S, et al. ESC Guidelines for the management of acute coronary syndromes in patients presenting without persistent ST-segment elevation: The Task Force for the management of acute coronary syndromes (ACS) in patients presenting without persistent ST-segment elevation of the European Society of Cardiology (ESC). Eur Heart J 2011;32:2999-3054.

5. Kumar A, Cannon CP. Acute coronary syndromes: diagnosis and management, part I. Mayo Clin Proc 2009;84:917-38.

6. Meikle PJ, Wong G, Tsorotes D, et al. Plasma lipidomic analysis of stable and unstable coronary artery disease. Arterioscler Thromb Vasc Biol 2011;31:2723-32.

7. Finn AV, Nakano M, Narula J, et al. Concept of vulnerable/unstable plaque. Arterioscler Thromb Vasc Biol 2010;30:1282-92.

8. Hannun YA, Obeid LM. Principles of bioactive lipid signalling: lessons from sphingolipids. Nat Rev Mol Cell Biol 2008;9:139-50.

9. Borodzicz S, Czarzasta K, Kuch M, et al. Sphingolipids in cardiovascular diseases and metabolic disorders. Lipids Health Dis 2015;14:55.

10. Schissel SL, Tweedie-Hardman J, Rapp JH, et al. Rabbit aorta and human atherosclerotic lesions hydrolyze the sphingomyelin of retained low-density lipoprotein. Proposed role for arterial-wall sphingomyelinase in subendothelial retention and aggregation of atherogenic lipoproteins. J Clin Invest 1996;98:1455-64.

11. Cheng JM, Suoniemi M, Kardys I, et al. Plasma concentrations of molecular lipid species in relation to coronary plaque characteristics and cardiovascular outcome: Results of the ATHEROREMO-IVUS study. Atherosclerosis 2015;243:560-6.

12. $\mathrm{Xu} \mathrm{J}$, Yeh $\mathrm{CH}$, Chen $\mathrm{S}$, et al. Involvement of de novo ceramide biosynthesis in tumor necrosis factor-alpha/cycloheximide-induced cerebral endothelial cell death. J Biol Chem 1998;273:16521-6.

13. Modur V, Zimmerman GA, Prescott SM, et al. Endothelial cell inflammatory responses to tumor necrosis factor alpha. Ceramidedependent and -independent mitogen-activated protein kinase cascades. J Biol Chem 1996;271:13094-102.

14. Sawada M, Kiyono T, Nakashima S, et al. Molecular mechanisms of TNF-alpha-induced ceramide formation in human glioma cells: P53mediated oxidant stress-dependent and -independent pathways. Cell Death Differ 2004;11:997-1008.

15. Augé N, Maupas-Schwalm F, Elbaz M, et al. Role for matrix metalloproteinase-2 in oxidized low-density lipoprotein-induced activation of the sphingomyelin/ceramide pathway and smooth muscle cell proliferation. Circulation 2004;110:571-8.

16. Spijkers LJ, van den Akker RF, Janssen BJ, et al. Hypertension is associated with marked alterations in sphingolipid biology: a potential role for ceramide. PLoS One 2011;6:e21817.

17. Huang $\mathrm{H}$, Kasumov $\mathrm{T}$, Gatmaitan $\mathrm{P}$, et al. Gastric bypass surgery reduces plasma ceramide subspecies and improves insulin sensitivity in severely obese patients. Obesity 2011;19:2235-40.

18. Haus JM, Kashyap SR, Kasumov T, et al. Plasma ceramides are elevated in obese subjects with type 2 diabetes and correlate with the severity of insulin resistance. Diabetes 2009;58:337-43.

19. Laaksonen R, Ekroos K, Sysi-Aho M, et al. Plasma ceramides predict cardiovascular death in patients with stable coronary artery disease and acute coronary syndromes beyond LDL-cholesterol. Eur Heart J 2016;37:1967-76.

20. Amsterdam EA, Wenger NK, Brindis RG, et al. 2014 AHAVACC guideline for the management of patients with non-ST-elevation acute coronary syndromes: executive summary: a report of the American College of Cardiology/American Heart Association Task Force on Practice Guidelines. Circulation 2014;130:2354-94.

21. Thygesen K, Alpert JS, Jaffe AS, et al. Third universal definition of myocardial infarction. J Am Coll Cardiol 2012;60:1581-98.

22. Tarasov K, Ekroos K, Suoniemi M, et al. Molecular lipids identify cardiovascular risk and are efficiently lowered by simvastatin and PCSK9 deficiency. J Clin Endocrinol Metab 2014;99:E45-E52.

23. Meeusen JW, Donato LJ, Bryant SC, et al. Plasma Ceramides: A Novel Predictor of Major Adverse Cardiovascular Events After Coronary Angiography. Arterioscler Thromb Vasc Biol 2018;38:1933-9.

24. Ambroise GJMAD. Analyzing Microarray Gene Expression Data. 2005.

25. Kuhn M, Johnson K. Applied Predictive Modeling. 2013.

26. James G, Witten D, Hastie T, et al. An Introduction to Statistical Learning. 2013.

27. Wang DD, Toledo E, Hruby A, et al. Plasma Ceramides, Mediterranean Diet, and Incident Cardiovascular Disease in the PREDIMED Trial (Prevención con Dieta Mediterránea). Circulation 2017;135:2028-40. 
28. Anroedh S, Hilvo M, Akkerhuis KM, et al. Plasma concentrations of molecular lipid species predict long-term clinical outcome in coronary artery disease patients. J Lipid Res 2018;59:1729-37.

29. de Carvalho LP, Tan SH, Ow GS, et al. Plasma ceramides as prognostic biomarkers and their arterial and myocardial tissue correlates in acute myocardial infarction. JACC Basic Trans/ Sci 2018;3:163-75.
30. Möckel M, Searle J, Hamm C, et al. Early discharge using single cardiac troponin and copeptin testing in patients with suspected acute coronary syndrome (ACS): a randomized, controlled clinical process study. Eur Heart J 2015;36:369-76.

31. de Lemos JA. Increasingly sensitive assays for cardiac troponins: a review. JAMA 2013;309:2262-9. 